\title{
Story Generation with Rich Details
}

\author{
Fangzhou Zhai $^{\dagger}$, Vera Demberg ${ }^{\dagger, \ddagger}$, and Alexander Koller ${ }^{\dagger}$ \\ ${ }^{\dagger}$ Dept. of Language Science and Technology \\ ${ }^{\ddagger}$ Dept. of Computer Science \\ Saarland Informatics Campus \\ Saarland University \\ \{fzhai, vera, koller\}@coli.uni-saarland.de
}

\begin{abstract}
Automatically generated stories should be not only coherent, but also interesting. Thus apart from realizing a story line, the text also have to include rich details to engage the readers. We propose a model that features two different generation components: (1) an outliner, which proceeds the main story line to establish global coherence; and (2) a detailer, which supplies relevant details to the story in a locally coherent manner. Human evaluation show that our model substantially improves the informativeness of generated text while retaining its coherence, outperforming a number of baselines.
\end{abstract}

\section{Introduction}

Story generation is the task of automatically crafting stories. Recent neural story generation systems have been able to produce coherent stories. Global coherence could be established by conditioning language models on the longer-term intention of the text. One could provide a topic (see, e.g. Fan et al. (2018), Fan et al. (2019)), a list of events (see, e.g. Zhai et al. (2019), Martin et al. (2018), Ammanabrolu et al. (2019)) or entities (see, e.g. Kiddon et al. (2016), Clark et al. (2018)) etc., to guide the generation process. But apart from being globally coherent, a story also needs to be interesting to engage its readers.

yesterday $i$ went grocery shopping . i made a list of my list and drove to the grocery store. when $i$ entered the store, $i$ grabbed a shopping cart and pushed the cart down to the meat aisle. $i$ got all my items, and crossed items on my list . $i$ went to the checkout register and paid for my groceries . i put my groceries in my cart and left .

Figure 1: A story about grocery shopping generated by Zhai et al. (2019), which is globally coherent but really boring.

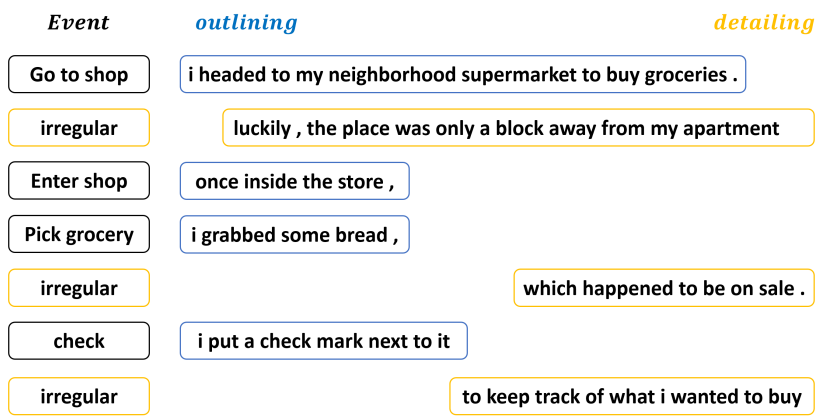

Figure 2: Sample Data from InSCRIPT. The left column list the event annotations, and on the right are the text segments corresponding to each event annotation. The story naturally decomposes to be an alternating effort between outlining and detailing.

Figure 1 shows a story about grocery shopping. The story is globally coherent as a narration of grocery shopping, but no one would be interested by such a story: it hardly provides any further information than what the topic grocery shopping already indicates.

Our key observation is that, story writing could be seen as the joint efforts of two different components, and a story generation system would benefit from modelling them differently. (1) Outlining, where a

This work is licensed under a Creative Commons Attribution 4.0 International Licence. Licence details: http:// creativecommons.org/licenses/by/4.0/. 
story realizes a (usually) linear story line step by step, thus establishing the global coherence of the text. (2) Detailing, where the author supplies details about some of the steps in the story line such that the story becomes informative and interests its reader. A neural story generation system that conditions the generation on the story line accomplishes the former but may well fail the latter.

We propose a model that generates stories with rich details, by addressing these two components differently: an outliner realizes consecutive events to establish the story line, whereas a detailer supplies additional details at specific points along the story line. Human evaluations show that our system significantly outperforms various baselines in terms of informativeness, its outputs maintaining their coherence as stories about daily activities.

\section{Model}

\subsection{Task and Data}

We work with INSCRIPT (Modi et al. (2016)), which contains around 100 stories for each of 10 daily activities like grocery shopping. Most of its verbal phrases are annotated with event types. There are two categories of events. (1) Regular events are closely related to the specific daily activity. These events correspond to the fundamental steps of the activity, like 'go to the shop, pick groceries, pay'. (2) Irregular events are not directly related to the core content of that activity, but instead supply relevant details (see figure 2). Regular events correspond to the outlining component, whereas irregular events correspond to the detailing component.

On average, there are 20 regular event types per activity. Events with labels 'unrelated (not related to the main activity), non-script (related but not a part of the activity per se), unknown' and other are considered irregular. Due to the varying nature of irregular events' content, they receive generic annotations. In total, the corpus contains $234 \mathrm{k}$ tokens, and realizes $15 \mathrm{k}$ event instances. About $40 \%$ of these events are irregular. We automatically dissect the stories to assign each event annotation to its corresponding text segment based on POS tags.

Our system receives a plausible sequence of events as input, which we term an agenda. Agendas are automatically generated by a bi-gram language model trained on the event sequences from the corpus; an agenda includes irregular event items, which specifies locations where additional details are needed. The model generates a story which realizes the agenda.

\subsection{Model Specifics}

Our neural model is an enriched ATT-SEQ2SEQ model with two different decoders: (1) an outliner, which generates regular segments to instantiate the regular event items in the agenda; and (2) a detailer, which generates irregular segments to supply details to the story. The model generates a story segment by segment, alternating between the decoders to address one event at a time. Thus technically, the neural part of the model receives (1) the input sequence, i.e. segments $s=\left\langle s_{1} \ldots s_{i-1}\right\rangle$ that are already generated and (2) the agenda $a=\left\langle e_{1} \ldots e_{n}\right\rangle$ that consists of $n$ events in total as input; as the output, it generates the next text segment $s_{i}$ which corresponds to the current event $e_{i}$.

Encoder We encode the input sequence in an agenda-aware manner:

$$
\boldsymbol{f}_{\boldsymbol{i}}=\operatorname{Enc}\left(\varphi_{w}\left(s_{<i}\right) ; \varphi_{e}\left(e_{<i}\right)\right)
$$

Here $\varphi_{w}(\cdot)$ denotes the word embeddings, which we initialize with pre-trained Glove embeddings (Pennington et al. (2014)); $\varphi_{e}(\cdot)$ is the event embeddings, which we initialize randomly. The embedding of each token in the history $s_{<i}$ is concatenated (;) with the embedding $\varphi_{e}\left(e_{i}\right)$ of its corresponding event $e_{i}$ so the encoding process is aware of the agenda. $\operatorname{Enc}(\cdot)$ is a single layer Bi-LSTM sequence encoder. The outcome $f_{i}$ is a list of vectors, each of which collects the features of its corresponding input token.

Outliner The outliner generates the text segment corresponding to the next event $e_{i}$ in the agenda, if it is regular. Here we use a Bi-LSTM sequence decoder which has access to a dot-product attention (Luong 
et al. (2015)) over the encoded sequence $f_{i}$. At each decoding step $t$ it yields a distribution from which one could sample the next token:

$$
p_{t}=\operatorname{Dec}\left(\varphi_{w}\left(\operatorname{tok}_{t-1}\right) ; \operatorname{att}\left(d_{t-1}, \boldsymbol{f}_{\boldsymbol{i}}\right) ; \varphi_{e}\left(e_{i}\right)\right)
$$

here, $d_{t-1}$ is the inner state of the decoder before generating the $t$-th token of the current segment; att $(\cdot, \cdot)$ denotes dot-product attention, so att $\left(d_{t-1}, \boldsymbol{f}_{\boldsymbol{i}}\right)$ collects information from the encoder; $t_{t-1}$ is the token generated in the previous step $t-1 ; \varphi_{e}\left(e_{i}\right)$ denotes the embedding of the target event. The complete segment will be generated with beam-search.

Detailer If the next event $e_{i}$ is irregular, the detailer generates its text segment. Whereas the outliner gets a regular event $e_{i}$ like pick up groceries which is informative of the content of the next segment, the detailer only gets an irregular event type, which incorporates little information. This is the main technical challenge of the paper, which we tackle with the following efforts.

(1) To select the content of the current segment, we condition the decoding process on its most important context: the previous regular event $e_{-}$and the successive regular event $e_{+}$. Thus at each decoding step $t$, it produces:

$$
q_{t}=\operatorname{Dec}\left(\varphi_{w}\left(t_{o k_{t-1}}\right) ; \operatorname{att}\left(d_{t-1}, \boldsymbol{f}_{\boldsymbol{i}}\right) ; \varphi_{e}\left(e_{-}\right) ; \varphi_{e}\left(e_{+}\right)\right)
$$

Here, $\operatorname{Dec}(\cdot)$ is once again a single layer Bi-LSTM.

(2) The detailer also adopts the maximum mutual information (MMI) objective. First used in conjunction with SEQ2SEQ models by Li et al. (2016), the technique promotes the generation of specific, meaningful texts by moderately suppressing generic language generations. The idea is, instead of maximizing data likelihood, one could maximize the mutual information $I(c, s)$ between the context $c$ and the generation $s$ to promote the correspondence between the two, thus improving the informativeness of the text. The MMI decoding objective generalizes to:

$$
s=\underset{s}{\arg \max }[\log (p(s \mid c))-\lambda \cdot \log (p(s))]
$$

which is the maximum likelihood objective minus a language model term, so it is also termed an anti-LM objective. In practice, equivalently, we follow the approach proposed by $\mathrm{Li}$ et al. (2016): we keep the maximum likelihood training intact, whereas in the inference phase, we use a pre-trained language model on INSCRIPT to estimate the anti-LM term, and add it to the scoring within beam search. The coefficient $\lambda$ is set at 0.1 .

Overall, our maximization objective is

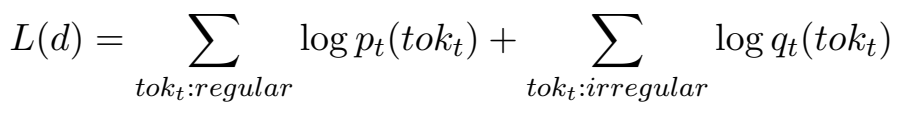

\subsection{Generation}

In the inference phase, we perform beam search to generate each segment. The beam size is set to 5 for the outliner. For the detailer, the scoring also exploits the anti-LM term, so a larger beam size is needed to effectively exploit the MMI objective, thus we set its beam size to 100 .

\section{Experiments}

\subsection{Set-up, Implementation and Optimization}

$5 \%$ of the stories in INSCRIPT are randomly selected as the validation set. As we use human evaluation, no test set is necessary. The model is implemented with AllenNLP 0.9.0 (Gardner et al. (2017)). Hyperparameters were chosen by random hyper parameter search (Bergstra and Bengio (2012)). The model is optimized with Adam (Kingma and $\mathrm{Ba}(2014)$ ) at a learning rate of $7.5 \times 10^{-4}$. Dimensions of the encoder and the decoder are both 256 . The word embedding size is fixed at 300 as we initialize it with the 840B version of the pre-trained Glove. A dropout (Srivastava et al. (2014)) at rate 0.69 is applied to 
all dense connections. Gradient norms are clipped at 2.0. We use early stopping with a patience of 35 to further regularize the training. Each training session takes on average 4 hours on a Tesla v100, whereas the generation speed is on average 8 tokens/second ${ }^{1}$.

\subsection{Evaluation}

Baselines We evaluate our model against three baselines: (1) the GRU-based model from Zhai et al. (2019), as it also takes an ordered agenda as input; (2) an ablated version of our model, SINGLE DECODER, which uses only one decoder, the outliner, to neutralize the effort to treat regular and irregular contents differently; (3) the human authored stories from INSCRIPT, to upper bound the performance. For some intuition, table 1 provides a few sample texts. Other external story generation systems we considered are not suitable either because they do not admit a compatible input format (i.e. taking a linear agenda as input) or that they cannot fully exploit the temporal order information encoded in an linear agenda (such systems tend to perform poorly on INSCRIPT based story generation (see Zhai et al. (2019)).

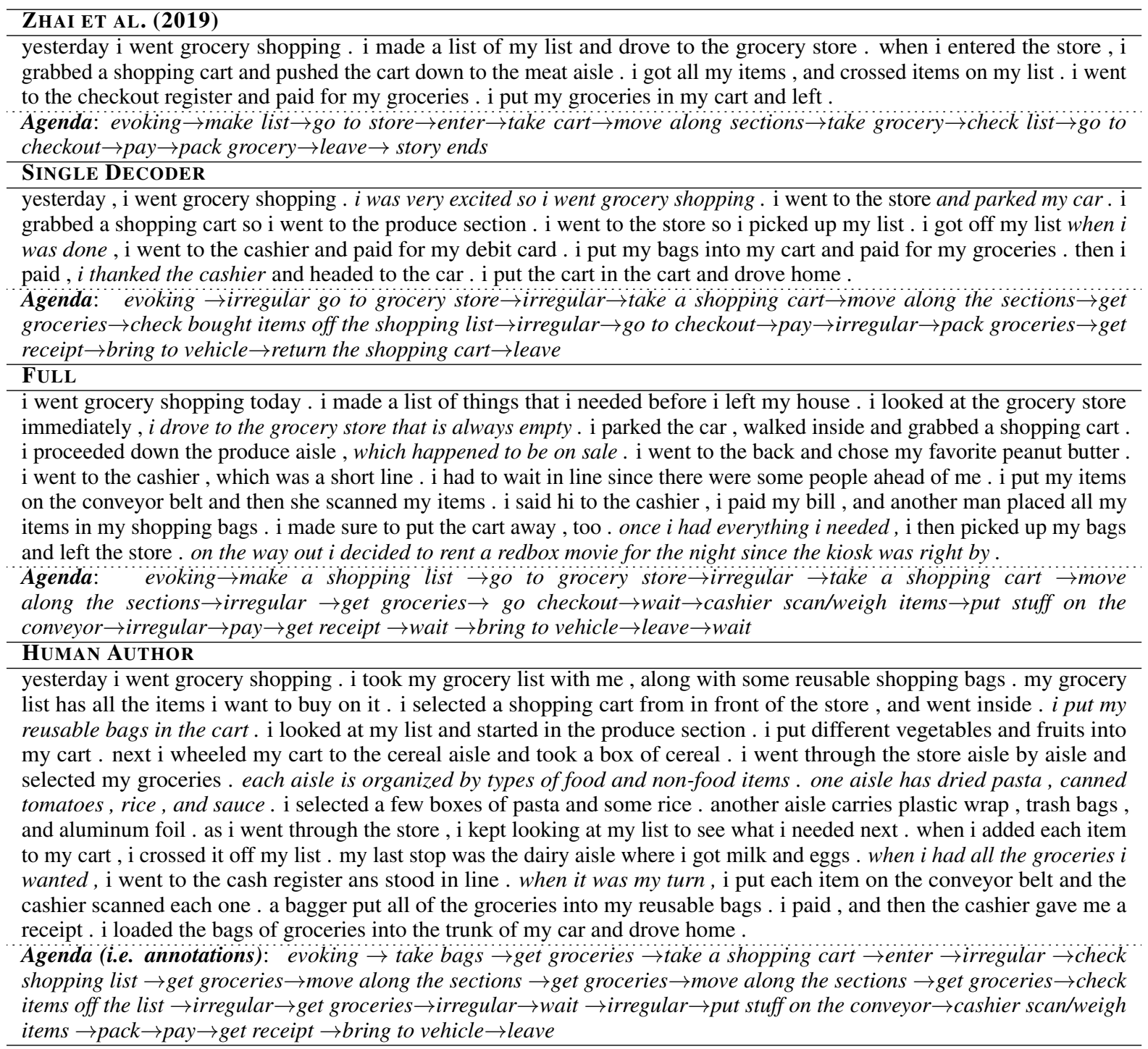

Table 1: Grocery shopping stories generated by different models, together with the agendas. The text corresponding to irregular events are italicized. We could see that the text produced by our model provides much richer details than the automatic baselines.

\footnotetext{
${ }^{1}$ For more details and interest in reproducing our results, the data and code could be found at https://github.com/ coli-saar/Story-Generation-with-Rich-Details.
} 
Experiment Design We evaluate the output text by crowd-sourcing. Our evaluation has two purposes. (1) As our main objective, we assess how much detail a story includes, i.e. how informative a story is about a specific experience. This is captured with an informativeness score.

(2) We want to make sure the improvement in informativeness does not sacrifice the stories' global coherence. Therefore, we assess whether a story is globally coherent wrt. the daily activity it is about, e.g. going grocery shopping. That means, a story should incorporate the common sense knowledge of going grocery shopping, including the necessary steps and their temporal order. This part involves five questions: syntax evaluates the basic syntax; global coherence evaluates the common sense knowledge about the activity included in the story; coverage evaluates whether the story realizes each event in the agenda (for a human-authored story, we take its event annotations as its agenda); relevance evaluates whether the story stays on-topic; local coherence evaluates the flow of successive sentences, in terms of both content and fluency. It also verifies whether the transition between two different decoders is smooth. Ideally, our model should establish as much global coherence as Zhai et al. (2019).

The evaluation experiment is implemented with LingoTurk (Pusse et al. (2016)) and conducted on Prolific (https://www.prolific.co/). The questions are presented as slide-bars. We evaluate 4 stories per scenario per system, and hire 10 native English speakers to score each story.

\begin{tabular}{|c|c|c|c|c|c|c|}
\hline & Coverage & Syntax & $\begin{array}{l}\text { Global } \\
\text { Coherence }\end{array}$ & $\begin{array}{l}\text { Local } \\
\text { Coherence }\end{array}$ & Relevance & Informativeness \\
\hline HUMAN AUTHOR & $76 \%^{f s z}$ & $.62^{f s z}$ & $.66^{f s z}$ & $.68^{f s z}$ & $.60^{f s z}$ & $.66^{f s z}$ \\
\hline FULL & $63 \%^{s^{\cdots}}$ & $43^{s^{\cdots}}$ & $.49^{\cdots}$ & $.42^{\cdots}$ & $.59^{\cdots}$ & $.51^{z \cdots}$ \\
\hline SINGLE DECODER & $57 \%$ & .38 & .42 & .38 & .52 & $.47^{z}$ \\
\hline ZHAI ET AL. (2019) & $66 \%$ fs & $.45^{s}$ & .40 & $.44^{s}$ & .54 & .38 \\
\hline
\end{tabular}

Table 2: Results from human evaluation. The range are all $[0,1]$.

Results The results are given in Table 2. First of all, we could see that on almost all metrics, HUMAN AUTHOR outperformed all other systems by a large margin, fulfilling its role as an upper bound. For our own model, we see that both requirements are met to validate its effectiveness: (1) it outperformed ZHAI ET AL. (2019) in informativeness significantly and by a large margin, indicating that the generated stories include much richer details about the specific experiences; (2) it performed comparably to ZHAI ET AL. (2019) on the first five metrics, which means it still contains the common sense knowledge of the respective daily activities, thus does not harm global coherence of the stories when trying to improve their informativeness. We also see that FULL outperformed SINGLE DECODER on all metrics, supporting our point that story writing decomposes into outlining and detailing, and that the modelling of the components should be addressed differently.

\section{Conclusion}

We seek to generate detail-rich stories in a coherent manner. We address the story generation process by a combined effort of two components: the outliner that proceeds the story, and the detailer that supplies context-relevant details. The system generates detail-rich stories while maintaining their global coherence.

\section{Acknowledgement}

The research is funded by the Deutsche Forschungsgemeinschaft (DFG, German Research Foundation) Project-ID 232722074 - SFB 1102. We thank our reviewers for their constructive feedback.

\section{References}

Prithviraj Ammanabrolu, Ethan Tien, Wesley Cheung, Zhaochen Luo, William Ma, Lara Martin, and Mark Riedl. 2019. Guided neural language generation for automated storytelling. In Proceedings of the Second Workshop on Storytelling, pages 46-55. 
James Bergstra and Yoshua Bengio. 2012. Random search for hyper-parameter optimization. Journal of Machine Learning Research, 13(Feb):281-305.

Elizabeth Clark, Yangfeng Ji, and Noah A Smith. 2018. Neural text generation in stories using entity representations as context. In Proceedings of the 2018 Conference of the North American Chapter of the Association for Computational Linguistics: Human Language Technologies, Volume 1 (Long Papers), pages 2250-2260.

Angela Fan, Mike Lewis, and Yann Dauphin. 2018. Hierarchical neural story generation. In Proceedings of the 56th Annual Meeting of the Association for Computational Linguistics (Volume 1: Long Papers), pages 889-898.

Angela Fan, Mike Lewis, and Yann Dauphin. 2019. Strategies for structuring story generation. In Proceedings of the 57th Annual Meeting of the Association for Computational Linguistics, pages 2650-2660.

Matt Gardner, Joel Grus, Mark Neumann, Oyvind Tafjord, Pradeep Dasigi, Nelson F. Liu, Matthew Peters, Michael Schmitz, and Luke S. Zettlemoyer. 2017. Allennlp: A deep semantic natural language processing platform.

Chloé Kiddon, Luke Zettlemoyer, and Yejin Choi. 2016. Globally coherent text generation with neural checklist models. In Proceedings of the 2016 Conference on Empirical Methods in Natural Language Processing, pages 329-339.

Diederik P Kingma and Jimmy Ba. 2014. Adam: A method for stochastic optimization. arXiv preprint arXiv: 1412.6980 .

Jiwei Li, Michel Galley, Chris Brockett, Jianfeng Gao, and Bill Dolan. 2016. A diversity-promoting objective function for neural conversation models. In Proceedings of the 2016 Conference of the North American Chapter of the Association for Computational Linguistics: Human Language Technologies, pages 110-119, San Diego, California, June. Association for Computational Linguistics.

Minh-Thang Luong, Hieu Pham, and Christopher D Manning. 2015. Effective approaches to attention-based neural machine translation. In Proceedings of the 2015 Conference on Empirical Methods in Natural Language Processing, pages 1412-1421.

Lara J Martin, Prithviraj Ammanabrolu, Xinyu Wang, William Hancock, Shruti Singh, Brent Harrison, and Mark O Riedl. 2018. Event representations for automated story generation with deep neural nets. In Thirty-Second AAAI Conference on Artificial Intelligence.

Ashutosh Modi, Tatjana Anikina, Simon Ostermann, and Manfred Pinkal. 2016. Inscript: Narrative texts annotated with script information. In Proceedings of the Tenth International Conference on Language Resources and Evaluation (LREC'16), pages 3485-3493.

Jeffrey Pennington, Richard Socher, and Christopher D Manning. 2014. Glove: Global vectors for word representation. In Proceedings of the 2014 conference on empirical methods in natural language processing (EMNLP), pages $1532-1543$.

Florian Pusse, Asad Sayeed, and Vera Demberg. 2016. Lingoturk: managing crowdsourced tasks for psycholinguistics. In Proceedings of the 2016 Conference of the North American Chapter of the Association for Computational Linguistics: Demonstrations, pages 57-61.

Nitish Srivastava, Geoffrey Hinton, Alex Krizhevsky, Ilya Sutskever, and Ruslan Salakhutdinov. 2014. Dropout: A simple way to prevent neural networks from overfitting. The Journal of Machine Learning Research, 15(1):1929-1958.

Fangzhou Zhai, Vera Demberg, Pavel Shkadzko, Wei Shi, and Asad Sayeed. 2019. A hybrid model for globally coherent story generation. In Proceedings of the Second Workshop on Storytelling, pages 34-45. 